\title{
GEOMETRIC AND ARITHMETIC SUBGROUPS OF THE GROTHENDIECK-TEICHMÜLLER GROUP
}

\author{
HideKAZu Furusho
}

\begin{abstract}
We compare two geometrically constructed subgroups $\mathbb{\Gamma}$ and $G T K$ of the Grothendieck-Teichmüller group $\widehat{G T}$ with an arithmetically constructed subgroup $G T A$ by deducing arithmetic properties from geometric ones. We show that the intersection of $\mathbb{\Gamma}$ and $G T K$ is contained in a certain modification of GTA.
\end{abstract}

\section{Introduction}

The Grothendieck-Teichmüller group $\widehat{G T}$ is a subgroup of the automorphism group Aut $\widehat{F_{2}}$ of the free pro-finite group of $\widehat{F_{2}}$ of rank 2 . It is parameterized by elements of $\widehat{\mathbf{Z}}^{\times} \times\left[\widehat{F_{2}}, \widehat{F_{2}}\right]$ and is defined by three relations (I), (II) and (III) (see $\S 1)$. It is a pro-finite group version of the pro-algebraic group $\underline{G T}$ [Dr]. In his study of Galois representations on fundamental groups, Y. Ihara showed that the absolute Galois group $G_{\mathbf{Q}}=\operatorname{Gal}(\overline{\mathbf{Q}} / \mathbf{Q})$ can be embedded into $\widehat{G T}$ in [Ih94]. It is still open whether $G_{\mathbf{Q}}$ is equal to $\widehat{G T}$ or not. But recently there have been discovered other several new-type relations satisfied by $G_{\mathbf{Q}}$ in $\widehat{G T}$ (see [Ih00], [LNS] and $[\mathrm{NT}])$. In this article, we show a certain relationship among them. In $\S 1$, we shall recall the definition of $\widehat{G T}$ and make a small remark (Proposition 1.3) which has been possibly unknown so far. $\S 2$ is a review of the definitions of three variants $\mathbb{\Gamma}([\mathrm{LNS}]), G T K([\mathrm{Ih} 00])$ and $G T A$ ([Ih00]) of $\widehat{G T}$. In $\S 3$, we introduce main results of the author's master's thesis $[\mathrm{F}]$, concerning on a relationship among defining equations of $\mathbb{\Gamma}, G T K$ and $G T A$. In $\S 4$, we give many lemmas to complete their proofs.

\section{Review of the definition of $\widehat{G T}$}

Let $\widehat{F_{2}}$ be the pro-finite free group of rank 2 with generators $x$ and $y$. We define $\widehat{G T}$ to be the set of pairs $(\lambda, f) \in \widehat{\mathbf{Z}}^{\times} \times{\widehat{F_{2}}}^{\prime}$ (where ${\widehat{F_{2}}}^{\prime}$ means the topological

Received June 25, 2002.

2000 Mathematics Subject Classification. Primary 14G32, Secondary 11S80. 
commutator subgroup $\left[\widehat{F_{2}}, \widehat{F_{2}}\right]$ of $\widehat{F_{2}}$ ) satisfying the following three relations:

$$
\left\{\begin{array}{l}
\text { (I) } f(x, y) f(y, x)=1 \\
\text { (II) } f(z, x) z^{m} f(y, z) y^{m} f(x, y) x^{m}=1 \\
\quad \text { for } m=\frac{1}{2}(\lambda-1), x y z=1 \quad((3 \text {-cycle relation })) \\
\text { (III) } f\left(x_{12}, x_{23}\right) f\left(x_{34}, x_{45}\right) f\left(x_{51}, x_{12}\right) f\left(x_{23}, x_{34}\right) f\left(x_{45}, x_{51}\right)=1 \quad \text { in } \hat{P}_{5}^{*} \\
\quad((5 \text {-cycle relation })) .
\end{array}\right.
$$

Here $\hat{P}_{n}^{*}$ is the pro-finite pure sphere braid group with $n$ strings and $x_{i, j}:=x_{i, j}^{(n)}$ $(1 \leqslant i, j \leqslant n)$ are its standard generators [Ih94]. For $f \in \widehat{F_{2}}$ and elements $\alpha, \beta$ of a pro-finite group $H, f(\alpha, \beta)$ stands for the image of $f$ by the homomorphism $\phi: \widehat{F_{2}} \rightarrow H$ defined by $\phi(x)=\alpha, \phi(y)=\beta$. An element $\sigma=(\lambda, f) \in \widehat{G T}$ induces an endomorphism of $\widehat{F_{2}}$ by $\sigma(x)=x^{\lambda}, \sigma(y)=f^{-1} y^{\lambda} f$, from which we get an embedding $\widehat{\widehat{G T}} \hookrightarrow$ End $\widehat{F_{2}}$ and in fact we can regard $\underline{\widehat{G T}}$ as a sub-monoid of End $\widehat{F_{2}}([\mathrm{Dr}])$.

Definition 1.1 ([Dr]). The Grothendieck-Teichmüller group $\widehat{G T}$ is the group of invertible elements of $\widehat{G T}$ :

$$
\widehat{G T}:=\left\{\sigma=(\lambda, f) \in \text { Aut } \widehat{F_{2}} \mid(\lambda, f) \text { satisfies }(\mathrm{I}) \sim(\mathrm{III}) .\right\} .
$$

Remark 1.2. The above 5-cycle relation (III) is different from the original relation of $\widehat{G T}([\mathrm{Dr}])$

$$
\begin{aligned}
(\mathrm{III})_{\mathrm{DR}} \quad f\left(x_{12}^{(4)}, x_{23}^{(4)} x_{24}^{(4)}\right) & f\left(x_{13}^{(4)} x_{23}^{(4)}, x_{34}^{(4)}\right) \\
= & f\left(x_{23}^{(4)}, x_{34}^{(4)}\right) f\left(x_{12}^{(4)} x_{13}^{(4)}, x_{24}^{(4)} x_{34}^{(4)}\right) f\left(x_{12}^{(4)}, x_{23}^{(4)}\right) \text { in } \hat{P}_{4}
\end{aligned}
$$

which appeared in $[\mathrm{NT}], \S 1$, where $\hat{P}_{n}(n \in \mathbf{N})$ stands for the pro-finite pure braid group with $n$-strings, and, by abuse of notation, we use the notation $x_{i j}^{(n)}$ (which was used previously to denote the standard generators of $\hat{P}_{n}^{*}$ ) to denote the standard generators of $\hat{P}_{n}$. We can easily show that (I)+(III) is equivalent to $(\mathrm{I})+(\mathrm{III})_{\mathrm{DR}}$.

Proposition 1.3. Relation (III) implies relation (I) .

Proof. Recall that we have a basic projection $p: \hat{P}_{5}^{*} \rightarrow \hat{P}_{4}^{*}$ which sends $x_{i, j}=$ $x_{i, j}^{(5)} \in \hat{P}_{5}^{*}$ to $x_{i, j}^{(4)} \in \hat{P}_{4}^{*}(1 \leqslant i, j \leqslant 4)$ and $x_{i, 5} \in \hat{P}_{5}^{*}$ to $1 \in \hat{P}_{4}^{*}(1 \leqslant i \leqslant 5)$. It is immediate to see that (III) implies (I) because

$$
\begin{aligned}
& p\left(f\left(x_{12}, x_{23}\right) f\left(x_{34}, x_{45}\right) f\left(x_{51}, x_{12}\right) f\left(x_{23}, x_{34}\right) f\left(x_{45}, x_{51}\right)\right) \\
& \quad=f\left(x_{12}^{(4)}, x_{23}^{(4)}\right) f\left(x_{23}^{(4)}, x_{34}^{(4)}\right)=f\left(x_{12}^{(4)}, x_{23}^{(4)}\right) f\left(x_{23}^{(4)}, x_{12}^{(4)}\right)
\end{aligned}
$$

and $\hat{P}_{4}^{*}$ is a free pro-finite group of rank 2 with generators $x_{12}^{(4)}$ and $x_{23}^{(4)}$.

Proposition 1.4. Relation (III) $\mathrm{DR}$ implies relation (I) . 
Proof. Recall that we have a basic projection $q: \hat{P}_{4} \rightarrow \hat{P}_{4}^{*}$ which sends $x_{i, j}=$ $x_{i, j}^{(4)} \in \hat{P}_{4}$ to $x_{i, j}^{(4)} \in \hat{P}_{4}^{*}(1 \leqslant i, j \leqslant 4)$. It is immediate to see that (III) $)_{\mathrm{DR}}$ implies (I) because

$$
\begin{gathered}
q\left(f\left(x_{12}^{(4)}, x_{23}^{(4)} x_{24}^{(4)}\right) f\left(x_{13}^{(4)} x_{23}^{(4)}, x_{34}^{(4)}\right)\right)= \\
\begin{aligned}
& q\left(f\left(x_{23}^{(4)}, x_{34}^{(4)}\right) f\left(x_{12}^{(4)} x_{13}^{(4)}, x_{24}^{(4)} x_{34}^{(4)}\right) f\left(x_{12}^{(4)}, x_{12}^{(4)}\right) f\left(x_{34}^{(4)}, x_{34}^{(4)}\right)=1 \text { in } \hat{P}_{4}^{*},\right. \\
&=f\left(x_{23}^{(4)}, x_{34}^{(4)}\right) f\left(x_{14}^{(4)},\right.\left.x_{14}^{(4)}\right) f\left(x_{12}^{(4)}, x_{23}^{(4)}\right) \\
&=f\left(x_{23}^{(4)}, x_{12}^{(4)}\right) f\left(x_{12}^{(4)}, x_{23}^{(4)}\right) \text { in } \hat{P}_{4}^{*}
\end{aligned}
\end{gathered}
$$

since $f \in \widehat{F}_{2}^{\prime}$. Therefore (III) $)_{\mathrm{DR}}$ implies (I).

Therefore we see that (III) is equivalent to (III) $)_{\mathrm{DR}}$ without assuming (I). The author thanks the referee for suggesting the possibility of validity of Proposition 1.4 .

With the $G_{\mathbf{Q}^{-}}$action on the geometric fundamental group $\pi_{1}\left(\mathbf{P}_{\overline{\mathbf{Q}}}^{1}-\{0,1, \infty\}, \overrightarrow{01}\right)$ of the projective line minus 3 points (with respect to a certain tangential base point $\overrightarrow{01}$ ), we associate a pro-finite group homomorphism $\varphi: G_{\mathbf{Q}} \rightarrow$ Aut $\widehat{F_{2}}$ (recall that $\left.\widehat{F_{2}} \simeq \pi_{1}\left(\mathbf{P}_{\overline{\mathbf{Q}}}^{1}-\{0,1, \infty\}, \overrightarrow{01}\right)\right)$. It follows from Belyı̆'s theorem [Be] that $\varphi$ is injective. In [Ih90] and [Ih94], it was shown that $\varphi\left(G_{\mathbf{Q}}\right)$ lies in $\widehat{G T}$. Now to determine whether $G_{\mathbf{Q}}$ is equal to $\widehat{G T}$ or not is a basic open problem, which is also related to a project posed by A. Grothendieck in [Gr]. Recently there have been discovered other new-type relations which $G_{\mathbf{Q}}$ satisfies in $\widehat{G T}$, for example $\left(\mathrm{I}^{\prime}\right),\left(\mathrm{II}^{\prime}\right),\left(\mathrm{III}^{\prime}\right),(\mathrm{IV}),\left(\mathrm{IV}^{\prime}\right),(\mathrm{V}),\left(\mathrm{A}_{n}\right),\left(\mathrm{K}_{n}\right)$ for $n=1,2,3, \ldots$ (see [Ih00], [LNS], [NS] and [NT]). Although they do not seem to be deduced from defining relations (I), (II) and (III) of $\widehat{G T}$, it has not been shown yet whether they are really new ones and whether they are enough to characterize $G_{\mathbf{Q}}$ as a subgroup of $\widehat{G T}$.

\section{Review of the definitions of $\mathbb{\Gamma}, G T K$ and $G T A$}

Three subgroups $\mathbb{I}, G T K$ and GTA of $\widehat{G T}$ were introduced in [Ih00] and [LNS]. The subgroup $\mathbb{\Gamma}$ (resp. GTK) was geometrically constructed by P. Lochak, H. Nakamura, and L. Schneps in [LNS] (resp. by Y. Ihara in [Ih00]). On the other hand, GTA was arithmetically constructed by Y. Ihara in [Ih00]. They all contain $G_{\mathbf{Q}}$, but it has not been known whether they are really proper subgroups of $\widehat{G T}$ and whether they are equal to $G_{\mathbf{Q}}$.

\section{1. $\mathbb{\Gamma}$.}

Definition 2.1 ([LNS]). The new Grothendieck-Teichmüller group $\mathbb{\Gamma}$ is the subset of $\widehat{G T}$ defined as follows:

$$
\mathbb{I}:=\{\sigma=(\lambda, f) \in \widehat{G T} \mid(\lambda, f) \text { satisfies (III') and (IV) below. }\} .
$$




$$
\begin{cases}\left(\mathrm{III}^{\prime}\right) & g\left(x_{45}, x_{51}\right) f\left(x_{12}, x_{23}\right) f\left(x_{34}, x_{45}\right)=f\left(\sigma_{1} \sigma_{3}, \sigma_{2}^{2}\right) \quad \text { in } \hat{B_{5}} \\ (\mathrm{IV}) & f\left(\sigma_{1}, \sigma_{2}^{4}\right)=\sigma_{2}^{-8 \Psi_{2}^{(0)}(\sigma)} f\left(\sigma_{1}^{2}, \sigma_{2}^{2}\right) \sigma_{1}^{-4 \Psi_{2}^{(0)}(\sigma)}\left(\sigma_{2} \sigma_{1}\right)^{6 \Psi_{2}^{(0)}(\sigma)} \text { in } \hat{B_{3} .}\end{cases}
$$

Here $g(x, y) \in \widehat{F_{2}}$ is the auxiliary parameter (depending on $\sigma \in \widehat{G T}$ ) satisfying $f(x, y)=g(y, x)^{-1} g(x, y)$, which was introduced in [LS]. For $n \in \mathbf{N}, \sigma_{i}(1 \leqslant i<$ $n)$ stand for standard generators of $\hat{B_{n}}([\mathrm{LNS}])$. For the definition of $\Psi_{2}^{(0)}(\sigma)^{\dagger}$, see $\S \S 2.2$. In [LNS] and [NS], it was shown that actually $\mathbb{\Gamma}$ forms a subgroup of $\widehat{G T}$. Note that (III') implies (III) ([NS]). These two relations (III') and (IV) just describe the condition for elements of $\widehat{G T}$ to act (as $G_{\mathbf{Q}}$ does) on all types of pro-finite Teichmüller modular groups in a certain consistent way (for more details, see $[\mathrm{LNS}])$.

2.2. GTK. For a natural number $n$, let $H_{n}$ be the index $n$ normal subgroup of $\widehat{F_{2}}$ which is freely generated by $n+1$ elements $x^{n}, y, x^{-1} y x, \cdots, x^{-(n-1)} y x^{n-1}$. For $\sigma=(\lambda, f) \in \widehat{G T}, f$ belongs to $H_{n}$, since $H_{n} \supset\left[\widehat{F_{2}}, \widehat{F_{2}}\right]$. In [Ih99], Ihara constructed the extended Kummer 1-cocycle $\Psi_{n}^{(0)}(\sigma)$ for $\sigma \in \widehat{G T}$, which is the image of $f$ by the continuous group homomorphism $H_{n} \rightarrow \widehat{\mathbf{Z}}$ defined by $x^{n} \mapsto 0$, $y \mapsto 1, x^{-j} y x^{j} \mapsto 0(1 \leqslant j<n)$. We remark that especially for $\sigma \in G_{\mathbf{Q}}, \Psi_{n}^{(0)}(\sigma)$ is the Kummer 1-cocycle which is characterized by $\sigma(\sqrt[k]{n})=\sqrt[k]{n} \zeta_{k}^{-\Psi_{n}^{(0)}(\sigma)}$ for $k \in \mathbf{N}$, where $\zeta_{k}=\exp \left(\frac{2 \pi i}{k}\right)$. Suppose that $\varphi_{n}: H_{n} \rightarrow \widehat{F_{2}}$ is the continuous group homomorphism defined by $x^{n} \mapsto x, y \mapsto y, x^{-j} y x^{j} \mapsto 1(1 \leqslant j<n)$. We note that $\varphi_{n}\left(H_{n m}\right) \subset H_{m}$ and $\varphi_{n m}=\varphi_{m} \circ\left(\left.\varphi_{n}\right|_{H_{n m}}\right)$ for $n, m \in \mathbf{N}$.

Definition 2.2 ([Ih00]). The Grothendieck-Teichmüller-Kummer group GTK is the subset of $\widehat{G T}$ defined as follows:

$$
\begin{aligned}
& G T K_{n}:=\left\{\sigma=(\lambda, f) \in \widehat{G T} \mid(\lambda, f) \text { satisfies }\left(\mathrm{K}_{n}\right) \text { below. }\right\} \\
& G T K:=\bigcap_{n \in \mathbf{N}} G T K_{n} \\
& \left(\mathrm{~K}_{n}\right) \quad \varphi_{n}(f)=y^{\Psi_{n}^{(0)}(\sigma)} f .
\end{aligned}
$$

It follows immediately from [Ih00], Proposition 1, that $G T K_{n}$ and $G T K$ actually form subgroups of $\widehat{G T}$. Relation $\left(\mathrm{K}_{n}\right)$ just describes the condition for elements of $\widehat{G T}$ to act (as $G_{\mathbf{Q}}$ does) on $H_{n}$ and $\widehat{F_{2}}$ consistently with two algebraic morphisms, the Kummer covering $\mathbf{P}_{\overline{\mathbf{Q}}}^{1}-\left\{0, \mu_{n}, \infty\right\} \rightarrow \mathbf{P}_{\overline{\mathbf{Q}}}^{1}-\{0,1, \infty\}$ defined by $t \mapsto t^{n}$ and the natural inclusion $\mathbf{P}_{\overline{\mathbf{Q}}}^{1}-\left\{0, \mu_{n}, \infty\right\} \hookrightarrow \mathbf{P}_{\overline{\mathbf{Q}}}^{1}-\{0,1, \infty\}$ defined by $t \mapsto t$ (for more details, see [Ih00]).

\footnotetext{
${ }^{\dagger}$ The 1-cocycles $\rho_{2}(\sigma)$ and $\rho_{3}(\sigma)$ studied in [LNS], [LS], [NS] and [NT] are equal to $-\Psi_{2}^{(0)}(\sigma)$ and $-\Psi_{3}^{(0)}(\sigma)$ respectively (see $[\mathrm{NT}]$ ).
} 
2.3. GTA. By the $\widehat{F}_{2}^{a b}\left(:=\widehat{F_{2}} / \widehat{F}_{2}^{\prime}\right)$-action on $\left({\widehat{F_{2}}}^{\prime}\right)^{a b}:={\widehat{F_{2}}}^{\prime} /\left[\widehat{F}_{2}^{\prime}, \widehat{F}_{2}^{\prime}\right]$ induced from the conjugation $n \mapsto f n f^{-1}$ for $n \in \widehat{F}_{2}^{\prime}$ and $f \in \widehat{F_{2}}$, we can regard $\left(\widehat{F}_{2}^{\prime}\right)^{a b}$ as a free $A_{2}\left(:=\widehat{\mathbf{Z}}\left[\left[\widehat{F}_{2} a b\right]\right]\right)$-module of rank 1 , generated by the class $\overline{[x, y]} \in\left({\widehat{F_{2}}}^{\prime}\right)^{a b}$ of $[x, y]:=x y x^{-1} y^{-1} \in{\widehat{F_{2}}}^{\prime}$ (for more details, see [Ih99]). Thus the action of $\sigma=(\lambda, f) \in \widehat{G T}$ on $\left(\widehat{F}_{2}^{\prime}\right)^{a b}$ induced from that on $\widehat{F_{2}}$ is expressed as $\sigma(\overline{[x, y]})=B_{\sigma}^{\prime} \cdot \overline{[x, y]}$, where $B_{\sigma}^{\prime} \in A_{2}^{\times}$. The adelic beta function $B_{\sigma} \in A_{2}^{\times}$ was defined by $B_{\sigma}^{\prime}=\frac{x^{\lambda}-1}{x-1} \frac{y^{\lambda}-1}{y-1} B_{\sigma}$ in [A(ii)] (for $\sigma \in G_{\mathbf{Q}}$ ) and [Ih99] (for $\sigma \in$ $\widehat{G T})$. By the embedding constructed by G. W. Anderson in $[\mathrm{A}(\mathrm{ii})], B_{\sigma}$ can be regarded as a function on $(\mathbf{Q} / \mathbf{Z})^{\oplus 2}$ valued in $\widehat{\mathbf{Z}} \otimes \mathbf{Q}^{a b}$, where $\mathbf{Q}^{a b}$ stands for the maximal abelian extension field over $\mathbf{Q}$. In [Ih99], Proposition 1.6.1, it was shown that the adelic beta function has much analogy with the classical beta function. Especially it is remarkable that by using the 5-cycle relation (III) Ihara showed that the adelic beta function $B_{\sigma}\left(s_{1}, s_{2}\right)\left(\sigma \in \widehat{G T},\left(s_{1}, s_{2}\right) \in(\mathbf{Q} / \mathbf{Z})^{\oplus 2}\right)$ can be split (but not uniquely) into the following product: $B_{\sigma}\left(s_{1}, s_{2}\right)=\frac{\Gamma_{\sigma}\left(s_{1}\right) \Gamma_{\sigma}\left(s_{2}\right)}{\Gamma_{\sigma}\left(s_{1}+s_{2}\right)}$. Here the adelic gamma function $\Gamma_{\sigma}$ is a function (uniquely determined up to a certain ambiguity) which is defined on $\mathbf{Q} / \mathbf{Z}$ and is valued in the product $\prod_{p \text { :prime }} \mathbb{W}\left(\overline{\mathbf{F}_{p}}\right)$ of the Witt vector ring $\mathbb{W}\left(\overline{\mathbf{F}_{p}}\right)$ of the algebraic closure of a finite field of characteristic $p$. In $[\mathrm{A}(\mathrm{ii})](\mathrm{i})$, Corollary 8.6.3, Anderson showed, as an analogy of Gauß' $n$-th multiplication formula of the classical gamma function, the $n$-th multiplication formula of the adelic gamma function $\Gamma_{\sigma}$ (for elements $\sigma$ of $\left.G_{\mathbf{Q}}\right)$ :

$$
\left(\mathrm{A}_{n}\right) \quad \prod_{n c=0} \frac{\Gamma_{\sigma}(s+c)}{\Gamma_{\sigma}(c)} \cdot \frac{1}{\Gamma_{\sigma}(n s)}=1 \otimes \exp \left[2 \pi i \cdot n \Psi_{n}^{(0)}(\sigma) s\right]
$$

by using Deligne's theory of absolute Hodge cycles. Ihara suggested that this arithmetic relation $\left(\mathrm{A}_{n}\right)$ could be a key condition to distinguish $G_{\mathbf{Q}}$ from $\widehat{G T}$ and considered the following new subgroup of $\widehat{G T}$ containing $G_{\mathbf{Q}}$.

Definition 2.3 ([Ih00]). The Grothendieck-Teichmüller-Anderson group GTA is the subset of $\widehat{G T}$ defined as follows:

$$
\begin{aligned}
& G T A_{n}:=\left\{\sigma=(\lambda, f) \in \widehat{G T} \mid \sigma \text { satisfies }\left(\mathrm{A}_{n}\right) .\right\} \\
& G T A:=\bigcap_{n \in \mathbf{N}} G T A_{n} .
\end{aligned}
$$

Remark 2.4. To state $\left(A_{n}\right)$ independently from $(\mathrm{I}) \sim(\mathrm{III})$, it is better to reformulate it as follows:

$$
\left(\mathrm{A}_{n}^{0}\right) \quad \prod_{0 \leqslant k \leqslant n-1} B_{\sigma}(s, k s) / \prod_{n c=0} B_{\sigma}(c, s)=1 \otimes \exp \left[2 \pi i \cdot n \Psi_{n}^{(0)}(\sigma) s\right]
$$

because the existence of $\Gamma_{\sigma}$ depends on $(\mathrm{I}) \sim(\mathrm{III})$. Here we use $\Gamma_{\sigma}(0)=1 \otimes 1$ ([Ih99], Proposition 1.7.1.(i)). 
It can be checked directly from the definitions of $\Psi_{n}^{(0)}(\sigma)$ and $\Gamma_{\sigma}(s)$ that $G T A$ and $G T A_{n}$ actually form subgroups of $\widehat{G T}$.

The relationship among the above three subgroups $\mathbb{\Gamma}, G T K$ and $G T A$ has not been fully understood yet. But we remark that it was shown in [Ih99] that relation $\left(\mathrm{K}_{n}\right)$ implies $\left(D \log \mathrm{A}_{n}\right)$, the logarithmic derivative of equation $\left(\mathrm{A}_{n}\right)$.

\section{Main results}

Theorem 3.1. The combination of relations (I), (II), (IV) and $\left(\mathrm{K}_{2}\right)$ implies relation $\left(\mathrm{A}_{2}^{0}\right)$.

Proof. At first, we introduce a new parameter $f_{+}$in the rank 3 free group $\widehat{F_{3}}$ with generators $W, X$ and $Y$. Recall that the index 2 subgroup $H_{2}(\S \S 2.2)$ of $\widehat{F_{2}}$ generated by $x y x^{-1}, x^{2}$ and $y$ can be identified with $\widehat{F_{3}}$ by sending $x y x^{-1}, x^{2}$, $y$ into $W, X, Y$ respectively. Since $y^{-2 \Psi_{2}^{(0)}(\sigma)} f$ lies on $H_{2}$, we obtain a unique element $f_{+}(W, X, Y)$ of $\widehat{F_{3}}$ such that

$$
y^{-2 \Psi_{2}^{(0)}(\sigma)} f(x, y)=f_{+}\left(x y x^{-1}, x^{2}, y\right) .
$$

It follows from [Ih99], Proposition 1.8.2 and Proposition 1.8.3, that the image

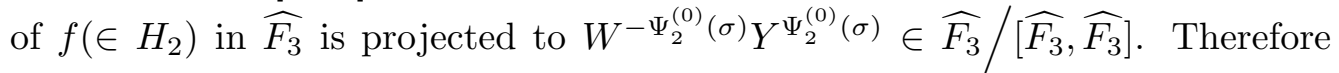
$W^{\Psi_{2}^{(0)}(\sigma)} Y^{\Psi_{2}^{(0)}(\sigma)} f_{+} \in\left[\widehat{F_{3}}, \widehat{F_{3}}\right]$. It is immediate that relation $\left(\mathrm{K}_{2}\right)$ is equivalent to

$$
y^{\Psi_{2}^{(0)}(\sigma)} f_{+}\left(x y x^{-1}, x^{2}, y\right)=f_{+}(1, x, y) .
$$

Here 1 stands for the unit element of $\widehat{F_{2}}$. In Lemma 4.1, we shall see that (IV) is re-expressed in terms of $f_{+} \in \widehat{F_{3}}$ as follows:

$$
f_{+}(W, X, Y) f_{+}\left(X^{-1} W^{-1} Y^{-1}, Y, X\right)=1 .
$$

In Lemma 4.2, we deduce the following equation from (I), (II) and (IV):

$$
\begin{aligned}
& Y^{m} f_{+}(W, X, Y) X^{m} f_{+}(Z, W, X) W^{m} f_{+}(Y, Z, W) \\
& Z^{m} f_{+}(X, Y, Z)=1 \text { for } W X Y Z=1
\end{aligned}
$$

Translating $\left(\mathrm{A}_{2}^{0}\right)$ into the terms of $\left.A_{2}=\widehat{\mathbf{Z}}\left[{\widehat{F_{2}}}^{a b}\right]\right]$ via Anderson's embedding (see $\S 2.3)$, we see that

$$
\left(\mathrm{A}_{2}^{0}\right) \quad \frac{B_{\sigma}(s, s)}{B_{\sigma}\left(\frac{1}{2}, s\right)}=1 \otimes \exp \left[2 \pi i \cdot \Psi_{2}^{(0)}(\sigma) s\right]
$$

is equivalent to

$$
B_{\sigma}\{\mathbf{x}, \mathbf{x}\}=\mathbf{x}^{-2 \Psi_{2}^{(0)}(\sigma)} B_{\sigma}\{-1, \mathbf{x}\} .
$$

Here $B_{\sigma}\{\mathbf{x}, \mathbf{x}\}$ (resp. $B_{\sigma}\{-1, \mathbf{x}\}$ ) stands for the image of $B_{\sigma} \in A_{2}$ by the map $A_{2} \rightarrow A_{2}$ induced from $\mathbf{x} \rightarrow \mathbf{x}, \mathbf{y} \rightarrow \mathbf{x}$ (resp. $\mathbf{x} \rightarrow-1, \mathbf{y} \rightarrow \mathbf{x}$ ), where boldfaces $\mathbf{x}, \mathbf{y}$ stand for the images in $A_{2}$ of standard generators $x, y$ of $\widehat{F_{2}}$. By properties 
[ii] [iv] of [Ih99], §2.3, which were deduced from (I) and (II), we see that saying $\left(\mathrm{A}_{2}^{0}\right)$ is equivalent to saying

$$
\frac{\mathbf{x}^{\lambda}+1}{\mathbf{x}^{m}(\mathbf{x}+1)} B_{\sigma}\left\{\mathbf{x}, \frac{1}{\mathbf{x}^{2}}\right\}=\mathbf{x}^{-2 \Psi_{2}^{(0)}(\sigma)} B_{\sigma}\{-1, \mathbf{x}\}
$$

It follows from [Ih99], Proposition 2.2.2, that saying $\left(\mathrm{A}_{2}^{0}\right)$ is equivalent to saying

$$
\frac{\mathbf{x}^{\lambda}+1}{\mathbf{x}^{m}(\mathbf{x}+1)}\left\{1-(\mathbf{x}-1) \frac{d f}{d x}\left(\mathbf{x}, \frac{1}{\mathbf{x}^{2}}\right)\right\}=\mathbf{x}^{-2 \Psi_{2}^{(0)}(\sigma)}\left\{1+2 \frac{d f}{d x}(-1, \mathbf{x})\right\} .
$$

Here we denote the image of $\frac{d f}{d x} \in \Lambda_{2}:=\widehat{\mathbf{Z}}\left[\left[\widehat{F_{2}}\right]\right]$ by the map $\Lambda_{2} \rightarrow A_{2}$ induced from $\mathbf{x} \mapsto-1, \mathbf{y} \mapsto \mathbf{x}$ by $\frac{d f}{d x}(-1, \mathbf{x})$ (for the definition of $\frac{d f}{d x} \in \Lambda_{2}$, see $\S 4$ ). For $\alpha \in \Lambda_{2}$ and $\mathbf{u}, \mathbf{v} \in{\widehat{F_{2}}}^{a b}$, we denote the image of $\alpha$ by the map $\Lambda_{2} \rightarrow A_{2}$ induced from $x \rightarrow \mathbf{u}, y \rightarrow \mathbf{v}$ by $\alpha(\mathbf{u}, \mathbf{v}) \in A_{2}$. By Lemma 4.3 , which will be deduced from (1), we see that the following is equivalent to $\left(\mathrm{A}_{2}^{0}\right)$.

$$
\begin{aligned}
& \frac{\mathbf{x}^{\lambda}+1}{\mathbf{x}^{m}(\mathbf{x}+1)} {\left[1-(\mathbf{x}-1)\left\{\mathbf{x}^{-2 \Psi_{2}^{(0)}(\sigma)} \frac{d f_{+}}{d W}\left(\frac{1}{\mathbf{x}^{2}}, \mathbf{x}^{2}, \frac{1}{\mathbf{x}^{2}}\right) \cdot\left(1-\frac{1}{\mathbf{x}^{2}}\right)\right.\right.} \\
&+\left.\left.\frac{d f_{+}}{d X}\left(\frac{1}{\mathbf{x}^{2}}, \mathbf{x}^{2}, \frac{1}{\mathbf{x}^{2}}\right) \cdot 2 \mathbf{x}\right\}\right] \\
&-\mathbf{x}^{-2 \Psi_{2}^{(0)}(\sigma)}\left[1+2 \mathbf{x}^{2 \Psi_{2}^{(0)}(\sigma)}\left\{(1-\mathbf{x}) \frac{d f_{+}}{d W}(\mathbf{x}, 1, \mathbf{x})-2 \frac{d f_{+}}{d X}(\mathbf{x}, 1, \mathbf{x})\right\}\right]=0 .
\end{aligned}
$$

For $\frac{d f_{+}}{d W}$ and $\frac{d f_{+}}{d X}$, see $\S 4$. By Lemma 4.4, which will be deduced from (2) (namely $\left.\left(\mathrm{K}_{2}\right)\right)$, we see that $\left(\mathrm{A}_{2}^{0}\right)$ is equivalent to

$$
\begin{aligned}
& \frac{\mathbf{x}^{\lambda}+1}{\mathbf{x}^{m}(\mathbf{x}+1)}\left\{1-\mathbf{x}^{-2 \Psi_{2}^{(0)}(\sigma)} \cdot(\mathbf{x}-1) \frac{d f_{+}}{d X}\left(1, \mathbf{x}, \frac{1}{\mathbf{x}^{2}}\right)\right\} \\
& -\mathbf{x}^{-2 \Psi_{2}^{(0)}(\sigma)}\left[1+2 \mathbf{x}_{2}^{2 \Psi_{2}^{(0)}(\sigma)}\left\{(1-\mathbf{x}) \frac{d f_{+}}{d W}(\mathbf{x}, 1, \mathbf{x})-2 \frac{d f_{+}}{d X}(\mathbf{x}, 1, \mathbf{x})\right\}\right]=0 .
\end{aligned}
$$

By formulae $(6) \sim(14)$ in $\S 4$, which will be deduced from (I), (II), (III) and $\left(\mathrm{K}_{2}\right)$, we get the following

$$
\begin{aligned}
\frac{d f_{+}}{d W}(\mathbf{x}, 1, \mathbf{x}) & =\mathbf{x}^{-2 \Psi_{2}^{(0)}-1} \cdot \frac{d f_{+}}{d W}\left(\frac{1}{\mathbf{x}^{2}}, \mathbf{x}, 1\right) \\
\frac{d f_{+}}{d X}(\mathbf{x}, 1, \mathbf{x}) & =0 \\
\frac{d f_{+}}{d X}\left(\frac{1}{\mathbf{x}^{2}}, \mathbf{x}, 1\right) & =\frac{\mathbf{x}+1}{\mathbf{x}^{2}} \frac{d f_{+}}{d W}\left(\frac{1}{\mathbf{x}^{2}}, \mathbf{x}, 1\right)+\frac{\mathbf{x}^{2 \Psi_{2}^{(0)}(\sigma)}-1}{\mathbf{x}-1} \\
\frac{d f_{+}}{d Y}\left(\mathbf{x}, \frac{1}{\mathbf{x}^{2}}, \mathbf{x}\right) & =-\mathbf{x}^{1-2 \Psi_{2}^{(0)}(\sigma)} \frac{d f_{+}}{d W}\left(1, \mathbf{x}, \frac{1}{\mathbf{x}^{2}}\right)-\frac{\mathbf{x}^{2 \Psi_{2}^{(0)}}(\sigma)}{\mathbf{x}^{2 \Psi_{2}^{(0)}(\sigma)}(\mathbf{x}-1)} \\
\frac{d f_{+}}{d Y}\left(\frac{1}{\mathbf{x}^{2}}, \mathbf{x}, 1\right) & =\frac{d f_{+}}{d W}\left(\frac{1}{\mathbf{x}^{2}}, \mathbf{x}, 1\right)
\end{aligned}
$$


By substituting $(W, X, Y)=\left(1, \mathbf{x}, \frac{1}{\mathbf{x}^{2}}\right)$ and above formulae to (15) in Lemma 4.6 , we obtain the validity of the equation (5), which means the validity of the Anderson duplication formula $\left(\mathrm{A}_{2}^{0}\right)$.

Therefore the combination of geometric relations (I), (II), (IV) and $\left(\mathrm{K}_{2}\right)$ implies arithmetic relation $\left(\mathrm{A}_{2}^{0}\right)$. Next we will consider three subgroups $\mathbb{\Gamma}, G T K$ and GTA.

Lemma 3.2. For $n, m \in \mathbf{N}$, the combination of relations $\left(\mathrm{A}_{n}\right)$ and $\left(\mathrm{A}_{m}\right)$ implies $\left(\mathrm{A}_{n m}\right)$.

Proof. Let $n \in \mathbf{N}$. By the slightly more detailed discussion on [Ih00], we find that relation $\left(\mathrm{A}_{n}\right)$ implies

$$
\Psi_{n a}^{(0)}(\sigma)=\Psi_{n}^{(0)}(\sigma)+\Psi_{a}^{(0)}(\sigma) \quad \text { for } \forall a \in \mathbf{N} .
$$

Therefore the combination of $\left(\mathrm{A}_{n}\right)$ and $\left(\mathrm{A}_{m}\right)$ implies the following:

$$
\begin{aligned}
\frac{\prod_{k=0}^{n m-1} \Gamma_{\sigma}\left(s+\frac{k}{n m}\right)}{\Gamma_{\sigma}(n m s) \prod_{k=0}^{n m-1} \Gamma_{\sigma}\left(\frac{k}{n m}\right)} & =1 \otimes \exp \left[2 \pi i \cdot n m\left\{\Psi_{n}^{(0)}(\sigma)+\Psi_{m}^{(0)}(\sigma)\right\} s\right] \\
& =1 \otimes \exp \left[2 \pi i \cdot n m \Psi_{n m}^{(0)}(\sigma) s\right]
\end{aligned}
$$

We also remark the following formulae, but it is not required to prove Theorem 3.4 below.

Lemma 3.3. For $n, m \in \mathbf{N}$, the combination of relations $\left(\mathrm{K}_{n}\right)$ and $\left(\mathrm{K}_{m}\right)$ implies $\left(\mathrm{K}_{n m}\right)$.

Proof. Let $n \in \mathbf{N}$. As in the same way of the case of Lemma 3.2, we get that relation $\left(\mathrm{K}_{n}\right)$ implies

$$
\Psi_{n a}^{(0)}(\sigma)=\Psi_{n}^{(0)}(\sigma)+\Psi_{a}^{(0)}(\sigma) \quad \text { for } \forall a \in \mathbf{N} .
$$

Therefore the combination of $\left(\mathrm{K}_{n}\right)$ and $\left(\mathrm{K}_{m}\right)$ implies the following:

$$
\begin{aligned}
\varphi_{n m}(f) & =\varphi_{n} \circ \varphi_{m}(f)=\varphi_{n}\left(y^{\Psi_{m}^{(0)}(\sigma)} f\right) \\
& =\varphi_{n}\left(y^{\Psi_{m}^{(0)}(\sigma)}\right) \varphi_{n}(f) y^{\Psi_{n}^{(0)}(\sigma)+\Psi_{m}^{(0)}(\sigma)} f=y^{\Psi_{n m}^{(0)}(\sigma)} f .
\end{aligned}
$$

Theorem 3.4. $G T K \cap \mathbb{\Gamma} \subseteq G T A_{2^{\infty}}$, where $G T A_{2^{\infty}}:=\bigcap_{n \in \mathbf{N}} G T A_{2^{n}}$.

Proof. We note that one relation $\left(\mathrm{A}_{2}\right)$ implies infinite ones $\left(\mathrm{A}_{2^{n}}\right)$ for $n=$ $1,2,3, \cdots$. Therefore, by combining Lemma 3.2 with Theorem 3.1 , we get the claim. 
Thus we get a relationship among the arithmetic subgroup $G T A_{2^{\infty}}$ and the geometric subgroups $G T K$ and $\mathbb{\Gamma}$. Relations (3) and (4) describe the $D_{4}$ (not $\left.D_{2}\right)$ symmetry of $\mathbf{P}^{1}-\{0, \pm 1, \infty\}$. Since they were essential in the proof of Theorem 3.1, it does not look possible, at least to the author, to deduce Anderson's $n$-th multiplication formula $(n \geqslant 5)$ from the $D_{n}$-symmetry of $\mathbf{P}^{1}-\left\{0, \mu_{n}, \infty\right\}$. Still we would expect the geometric interpretation of arithmetic Anderson's multiplication formulae which is originally of arithmetic nature to be a key to distinguish $G_{\mathbf{Q}}$ from $\widehat{G T}$.

\section{Miscellaneous lemmas}

We present auxiliary lemmas which were required in the proof of Theorem 3.1. Here we follow the notation in [Ih99].

Lemma 4.1. Relation (IV) is equivalent to (3).

Proof. Let $\widehat{F_{2}} /\left\langle\left\langle z^{2}\right\rangle\right\rangle$ denote the quotient pro-finite group of $\widehat{F_{2}}$ by the normal closure $\left\langle\left\langle z^{2}\right\rangle\right\rangle$ of $z^{2}=(x y)^{-2}$. By [NS], Theorem 2.2. (22),

$$
\begin{array}{rlrl}
(\mathrm{IV}) & \Longleftrightarrow y^{4 \Psi_{2}^{(0)}(\sigma)} f\left(x, y^{2}\right) x^{4 \Psi_{2}^{(0)}(\sigma)} f\left(y, x^{2}\right) \equiv 1 & \text { in } \widehat{F_{2}} /\left\langle\left\langle z^{2}\right\rangle\right\rangle \\
& \Longleftrightarrow f_{+}\left(x y^{2} x^{-1}, x^{2}, y^{2}\right) f_{+}\left(y x^{2} y^{-1}, y^{2}, x^{2}\right) \equiv 1 & \text { in } & \widehat{F_{2}} /\left\langle\left\langle z^{2}\right\rangle\right\rangle \\
& \Longleftrightarrow f_{+}\left(x y^{2} x^{-1}, x^{2}, y^{2}\right) f_{+}\left(x^{-1} y^{-2} x^{-1} y^{-2}, y^{2}, x^{2}\right) \equiv 1 & \text { in } & \widehat{F_{2}} /\left\langle\left\langle z^{2}\right\rangle\right\rangle .
\end{array}
$$

On the other hand, since $x y^{2} x^{-1}, x^{2}$ and $y^{2}$ generate a free pro-finite subgroup of rank 3 in $\widehat{F_{2}}$,

$$
(3) \Longleftrightarrow f_{+}\left(x y^{2} x^{-1}, x^{2}, y^{2}\right) f_{+}\left(x^{-1} y^{-2} x^{-1} y^{-2}, y^{2}, x^{2}\right)=1 \quad \text { in } \widehat{F_{2}} .
$$

From the argument in the proof of [NS], Theorem 2.2, the quotient classes of $x$, $y^{2}$ in $\widehat{F_{2}} /\left\langle\left\langle z^{2}\right\rangle\right\rangle$ generate a free pro-finite subgroup of rank 2 . Thus the images of $x y^{2} x^{-1}, x^{2}, y^{2}$ in $\widehat{F_{2}} /\left\langle\left\langle z^{2}\right\rangle\right\rangle$ generate a free pro-finite subgroup of rank 3 , from which it follows that (IV) is equivalent to (3).

Lemma 4.2. The combination of relations (I), (II) and (IV) implies (4).

Proof. By the permutation $x \mapsto y, y \mapsto x, z \mapsto x^{-1} y^{-1}$, (II) is re-expressed as follows:

$$
f(y, x) y^{m+1} f(z, y) z^{m+1} f(x, z) x^{m+1}=1 .
$$

By combining this with (II) and applying (I), we get

$$
x^{2 m+1} f(x, z)^{-1} z^{m} f(y, z) y^{2 m+1} f(y, z)^{-1} z^{m+1} f(x, z)=1 .
$$

Taking the image of the last equation by the continuous homomorphism induced from $x \mapsto y^{-1} x^{-1}, y \mapsto x, z \mapsto y$, we get

$$
\begin{gathered}
\left(y^{-1} x^{-1}\right)^{2 m+1} f_{+}\left(y^{-1} x^{-1} y x y, y^{-1} x^{-1} y^{-1} x^{-1}, y\right)^{-1} y^{m} f_{+}\left(x y x^{-1}, x^{2}, y\right) x^{2 m+1} \\
f_{+}\left(x y x^{-1}, x^{2}, y\right)^{-1} y^{m+1} f_{+}\left(y^{-1} x^{-1} y x y, y^{-1} x^{-1} y^{-1} x^{-1}, y\right)=1 .
\end{gathered}
$$


By (IV), or equivalently, by (3),

$$
\begin{aligned}
& \left(y^{-1} x^{-1} y^{-1} x^{-1}\right)^{m} \cdot f_{+}\left(x^{2}, y, y^{-1} x^{-1} y^{-1} x^{-1}\right) \cdot y^{m} \cdot f_{+}\left(x y x^{-1}, x^{2}, y\right) \cdot\left(x^{2}\right)^{m} \\
& f_{+}\left(y^{-1} x^{-1} y^{-1} x^{-1}, x y x^{-1}, x^{2}\right) \cdot\left(x y x^{-1}\right)^{m} \cdot f_{+}\left(y, y^{-1} x^{-1} y^{-1} x^{-1}, x y x^{-1}\right)=1 .
\end{aligned}
$$

Since the subgroup generated by $x^{2}, y$ and $y^{-1} x^{-1} y^{-1} x^{-1}$ is equal to the one generated by $x^{2}, y$ and $x y x^{-1}$, it is a free pro-finite subgroup of rank 3 in $\widehat{F_{2}}$, which implies (4).

By Anderson's theorem (see [Ih99], Theorem A.1.), the element $f \in \widehat{F_{2}}$ can be written uniquely in $\Lambda_{2}:=\widehat{\mathbf{Z}}\left[\left[\widehat{F}_{2}\right]\right]$ as follows:

$$
f(x, y)=1+\frac{d f}{d x} \cdot(x-1)+\frac{d f}{d y} \cdot(y-1)
$$

Put $\Lambda_{3}:=\widehat{\mathbf{Z}}\left[\left[\widehat{F_{3}}\right]\right]$. Similarly the element $f_{+} \in \widehat{F_{3}}=\langle W, X, Y\rangle^{\wedge}$ in $\S 3$ can be written uniquely in $\Lambda_{3}$ as follows:

$$
f_{+}(W, X, Y)=1+\frac{d f_{+}}{d W} \cdot(W-1)+\frac{d f_{+}}{d X} \cdot(X-1)+\frac{d f_{+}}{d Y} \cdot(Y-1)
$$

where $\frac{d f_{+}}{d W}, \frac{d f_{+}}{d X}, \frac{d f_{+}}{d Y} \in \Lambda_{3}$.

Lemma 4.3. Let $\sigma=(\lambda, f) \in \widehat{G T}$ and let $f_{+} \in \widehat{F_{3}}$ be as in (1). Then we have the following:

$$
\begin{aligned}
\frac{d f}{d x}(x, y)= & y^{2 \Psi_{2}^{(0)}(\sigma)} \frac{d f_{+}}{d W}\left(x y x^{-1}, x^{2}, y\right) \cdot\left(1-x y x^{-1}\right) \\
& +\frac{d f_{+}}{d X}\left(x y x^{-1}, x^{2}, y\right) \cdot 2 x \\
\frac{d f}{d y}(x, y)= & \frac{y^{2 \Psi_{2}^{(0)}(\sigma)}-1}{y-1}+y^{2 \Psi_{2}^{(0)}(\sigma)}\left\{\frac{d f_{+}}{d W}\left(x y x^{-1}, x^{2}, y\right) \cdot x\right. \\
& \left.+\frac{d f_{+}}{d Y}\left(x y x^{-1}, x^{2}, y\right)\right\} .
\end{aligned}
$$

Here for $\beta \in \Lambda_{3}$ and $a, b, c \in \widehat{F_{2}}$, we denote the image of $\beta$ by the map $\Lambda_{3} \rightarrow \Lambda_{2}$ induced from $W \mapsto a, X \mapsto b, Y \mapsto c$ by $\beta(a, b, c) \in \Lambda_{2}$.

Proof. It follows from (1) by a direct calculation. 
Lemma 4.4. Suppose that $\sigma=(\lambda, f) \in \widehat{G T}$ satisfies $\left(\mathrm{K}_{2}\right)$ and let $f_{+} \in \widehat{F_{3}}$ be as in (1). Then we have the following:

$$
\begin{aligned}
\frac{d f_{+}}{d X}(1, x, y) & =y^{\Psi_{2}^{(0)}(\sigma)} \frac{d f_{+}}{d W}\left(x y x^{-1}, x^{2}, y\right) \cdot\left(1-x y x^{-1}\right) \\
& +y^{-\Psi_{2}^{(0)}(\sigma)} \frac{d f_{+}}{d X}\left(x y x^{-1}, x^{2}, y\right) \cdot 2 x \\
\frac{d f_{+}}{d Y}(1, x, y) & =\frac{y^{\Psi_{2}^{(0)}(\sigma)}-1}{y-1}+y^{\Psi_{2}^{(0)}(\sigma)}\left\{\frac{d f_{+}}{d W}\left(x y x^{-1}, x^{2}, y\right) \cdot x\right. \\
& \left.+\frac{d f_{+}}{d Y}\left(x y x^{-1}, x^{2}, y\right)\right\} .
\end{aligned}
$$

Proof. It follows from (2) by a direct calculation.

Lemma 4.5. Suppose that $\sigma=(\lambda, f) \in \widehat{G T}$ satisfies $(I V)$ and let $f_{+} \in \widehat{F_{3}}$ be as in (1). Then we have the following:

$$
\begin{aligned}
\frac{d f_{+}}{d W}(W, X, Y)= & f_{+}(W, X, Y) \frac{d f_{+}}{d W}\left(X^{-1} W^{-1} Y^{-1}, Y, X\right) X^{-1} W^{-1}, \\
\frac{d f_{+}}{d X}(W, X, Y)= & f_{+}(W, X, Y)\left\{\frac{d f_{+}}{d W}\left(X^{-1} W^{-1} Y^{-1}, Y, X\right) \cdot X^{-1}\right. \\
& \left.-\frac{d f_{+}}{d Y}\left(X^{-1} W^{-1} Y^{-1}, Y, X\right)\right\}, \\
\frac{d f_{+}}{d Y}(W, X, Y)= & f_{+}(W, X, Y)\left\{\frac{d f_{+}}{d W}\left(X^{-1} W^{-1} Y^{-1}, Y, X\right) \cdot\right. \\
& \left.X^{-1} W^{-1} Y^{-1}+\frac{d f_{+}}{d X}\left(X^{-1} W^{-1} Y^{-1}, Y, X\right)\right\} .
\end{aligned}
$$

Proof. It follows from (3) by a direct calculation.

Lemma 4.6. Suppose that $\sigma=(\lambda, f) \in \widehat{G T}$ satisfies (IV) and let $f_{+} \in \widehat{F_{3}}$ be as in (1). Then we have the following:

$$
\begin{aligned}
Y^{m} & \frac{d f_{+}}{d W}(W, X, Y) \\
+ & Y^{m} f_{+}(W, X, Y) X^{m}\left\{\frac{d f_{+}}{d W}(Z, W, X) \cdot(-Z)+\frac{d f_{+}}{d X}(Z, W, X)\right\} \\
+ & Y^{m} f_{+}(W, X, Y) X^{m} f_{+}(Z, W, X) \frac{W^{m}-1}{W-1} \\
+ & Y^{m} f_{+}(W, X, Y) X^{m} f_{+}(Z, W, X) W^{m} \cdot \\
& \left\{\frac{d f_{+}}{d X}(Y, Z, W) \cdot(-Z)+\frac{d f_{+}}{d Y}(Y, Z, W)\right\} \\
+ & Y^{m} f_{+}(W, X, Y) X^{m} f_{+}(Z, W, X) W^{m} f_{+}(Y, Z, W) \frac{Z^{m}-1}{Z-1} \cdot(-Z) \\
+ & Y^{m} f_{+}(W, X, Y) X^{m} f_{+}(Z, W, X) W^{m} f_{+}(Y, Z, W) Z^{m} \cdot \\
& \left\{\frac{d f_{+}}{d Y}(X, Y, Z) \cdot(-Z)\right\}=0 \quad \text { for } \quad W X Y Z=1 .
\end{aligned}
$$


Proof. It follows from (4) by a direct calculation.

\section{Acknowledgement}

The author is profoundly grateful to my master thesis advisor Professor Y. Ihara for warmful encouragements. He also express his special thanks to H. Nakamura and H. Tsunogai for useful discussion and their support and to Professor A. Tamagawa for his kind advises. He is supported by JSPS Research Fellowships for Young Scientists.

\section{References}

[A(i)] G.W. Anderson, The hyperadelic gamma function: a précis, Galois representations and arithmetic algebraic geometry (Kyoto, 1985/Tokyo, 1986), 1-19, Adv. Stud. Pure Math., 12, North-Holland, Amsterdam, 1987.

[A(ii)] , The hyperadelic gamma function, Invent. Math. 95 (1989), 63-131.

[Be] G. V. Belyı̆, Galois extensions of a maximal cyclotomic field, (Russian) Izv. Akad. Nauk SSSR Ser. Mat. 43 (1979), 267-276, 479.

[Dr] V. G. Drinfel'd, On quasitriangular quasi-Hopf algebras and a group closely connected with $\operatorname{Gal}(\bar{Q} / Q)$, Leningrad Math. J. 2 (1991), 829-860.

[F] H. Furusho, Geometric and arithmetic subgroups of the Grothendieck-Teichmüller group, Master's thesis (in Japanese), RIMS, Kyoto University, March 2000.

[Gr] A. Grothendieck, Esquisse d'un programme, London Math. Soc. Lecture Note Ser., 242, Geometric Galois actions, 1, 5-48, Cambridge Univ. Press, Cambridge, 1997.

[Ih90] Y. Ihara, Braids, Galois groups, and some arithmetic functions, Proceedings of the International Congress of Mathematicians, Vol. I, II (Kyoto, 1990), 99-120, Math. Soc. Japan, Tokyo, 1991.

[Ih91] _ Automorphisms of pure sphere braid groups and Galois representations, The Grothendieck Festschrift, Vol. II, 353-373, Progr. Math., 87, Birkhäuser Boston, Boston, MA, 1990.

[Ih94] , On the embedding of $\operatorname{Gal}(\bar{Q} / Q)$ into $\widehat{\mathrm{GT}}$, London Math. Soc. Lecture Note Ser., 200, The Grothendieck theory of dessins d'enfants (Luminy, 1993), 289-321, Cambridge Univ. Press, Cambridge, 1994.

[Ih99] _ On beta and gamma functions associated with the Grothendieck-Teichmüller groups, Aspects of Galois theory (Gainesville, FL, 1996), 144-179, London Math. Soc. Lecture Note Ser., 256, Cambridge Univ. Press, Cambridge, 1999.

[Ih00] - On beta and gamma functions associated with the Grothendieck-Teichmüller group. II, J. Reine Angew. Math. 527 (2000), 1-11.

[LNS] P. Lochak, H. Nakamura, L. Schneps, On a new version of the GrothendieckTeichmüller group, C. R. Acad. Sci. Paris Sér. I Math. 325 (1997), 11-16.

[LS] P. Lochak, L. Schneps, A cohomological interpretation of the Grothendieck-Teichmüller group, Invent. Math. 127 (1997), 571-600.

[NS] H. Nakamura, L. Schneps, On a subgroup of the Grothendieck-Teichmüller group acting on the tower of profinite Teichmüller modular groups, Invent. Math. 141 (2000), 503560 .

[NT] H. Nakamura, H. Tsunogai, Harmonic and equianharmonic equations in the Grothendieck-Teichmüller group, to appear in Forum Math.

Research Institute for Mathematical Sciences Kyoto University, Kyoto 606-8502, JAPAN.

E-mail address: furusho@kurims.kyoto-u.ac.jp 